\title{
Superconductivity of Bi-III phase of elemental bismuth: Insights from muon-spin rotation and density functional theory
}

\author{
Rustem Khasanov, ${ }^{1, *}$ Hubertus Luetkens, ${ }^{1}$ Elvezio Morenzoni, ${ }^{1}$ Gediminas Simutis, ${ }^{1}$ Stephan Schönecker, ${ }^{2}$ Andreas Östlin, ${ }^{3}$ \\ Liviu Chioncel, ${ }^{3}$ and Alex Amato ${ }^{1}$ \\ ${ }^{1}$ Laboratory for Muon Spin Spectroscopy, Paul Scherrer Institute, CH-5232 Villigen PSI, Switzerland \\ ${ }^{2}$ Applied Materials Physics, Department of Materials Science and Engineering, KTH Royal Institute of Technology, \\ SE-10044 Stockholm, Sweden \\ ${ }^{3}$ Augsburg Center for Innovative Technologies, and Center for Electronic Correlations and Magnetism, Theoretical Physics III, \\ Institute of Physics, University of Augsburg, D-86135 Augsburg, Germany
}

(Received 20 February 2018; revised manuscript received 27 September 2018; published 12 October 2018)

\begin{abstract}
Using muon-spin rotation the pressure-induced superconductivity in the Bi-III phase of elemental bismuth (transition temperature $T_{\mathrm{c}} \simeq 7.05 \mathrm{~K}$ ) was investigated. A Ginzburg-Landau parameter $\kappa=\lambda / \xi=30(6)(\lambda$ is the magnetic penetration depth, $\xi$ is the coherence length) was estimated, which turns out to be the highest among known single element superconductors. The temperature dependence of the superconducting energy gap $[\Delta(T)]$ reconstructed from $\lambda^{-2}(T)$ deviates from the weakly coupled BCS prediction. The coupling strength $2 \Delta / k_{\mathrm{B}} T_{\mathrm{c}} \simeq$ 4.34 was estimated, thus implying that Bi-III stays within the strong-coupling regime. The density functional theory calculations suggest that superconductivity in Bi-III could be described within the Eliashberg approach with a characteristic phonon frequency $\omega_{\mathrm{ln}} \simeq 5.5 \mathrm{meV}$. An alternative pairing mechanism to the electron-phonon coupling involves the possibility of Cooper pairing induced by Fermi-surface nesting.
\end{abstract}

DOI: 10.1103/PhysRevB.98.140504

Following the intense research on superconductivity in cuprates and Fe-based superconductors, simpler materials such as elementary or binary compounds have attracted renewed interest. Recent results have shown that in spite of their simple composition they represent a playground for the discovery of new phenomena and unconventional characteristics. Binary compounds were reported to superconduct at surprisingly high critical temperatures $\left(T_{\mathrm{c}}\right.$ 's) reaching $\simeq 40 \mathrm{~K}$ for $\mathrm{MgB}_{2}$ [1], as well as the highest ever obtained $T_{\mathrm{c}}$ of $\sim 200 \mathrm{~K} \mathrm{in} \mathrm{SH}_{3}$ at an (extreme) pressure of $p \simeq 200 \mathrm{GPa}$ [2]. The basic elements with high $T_{\mathrm{c}}$ 's include $\mathrm{Li}\left(T_{\mathrm{c}} \approx 15-20 \mathrm{~K}\right.$ at $30 \mathrm{GPa})$ [3-5], $\mathrm{Ca}\left(T_{\mathrm{c}} \approx 21-29 \mathrm{~K}\right.$ at $\left.220 \mathrm{GPa}\right)$ [6], $\mathrm{Sc}$ and $\mathrm{Y}\left(T_{\mathrm{c}} \approx 20 \mathrm{~K}\right.$ near $\left.100 \mathrm{GPa}\right)[7,8], \mathrm{V}\left(T_{\mathrm{c}} \approx 17 \mathrm{~K}\right.$ at $120 \mathrm{GPa})$ [9,10], and $\mathrm{S}\left(T_{\mathrm{c}} \approx 17 \mathrm{~K}\right.$ at $\left.220 \mathrm{GPa}\right)$ [11]. Such high transition temperatures of basic elements indicate the importance of pressure as a tuning parameter of superconducting properties and its role in unraveling the intrinsic properties of the electronic system.

Among single element superconductors the Bi-III phase of elemental bismuth is one of the most interesting systems to study. Following Refs. [12-19], bismuth converts into a phase Bi-III, exhibiting superconductivity at $T_{\mathrm{c}} \simeq 7 \mathrm{~K}$, above a pressure of $\simeq 2.7 \mathrm{GPa}$. The crystal structure of Bi-III was resolved only recently (in the year 2000) and it was indexed as an incommensurate host-guest lattice [20]. It consists of two interpenetrating structures, with "guest" atoms forming chains within cylindrical cavities in the "host" lattice. In the $a b$ plane, the unit cells of guests and hosts match, while the ratio along the $c$-axis lattice parameters is incommensurate,

*rustem.khasanov@psi.ch $c_{\text {host }} / c_{\text {guest }}=1.309[20,21]$. Remarkably, this incommensurate structure may give rise to an additional acoustic mode, in the phonon spectrum, at very low frequencies arising from the sliding of one structure through the other, a process which has almost no energy cost [22-24]. Following Refs. [18,19], in Bi-III such a mode was suggested to be responsible for a very high electron-phonon coupling strength and gave rise to an enhanced transition temperature and a high upper critical field $\left(H_{\mathrm{c} 2}\right)$. It is worth noting, however, that the number of physical quantities studied so far for the Bi-III phase of elemental bismuth were mostly limited to $T_{\mathrm{c}}$ and $H_{\mathrm{c} 2}$ [12-19], which may not be enough to draw unambiguous conclusions on the type of superconducting mechanism.

In this Rapid Communication, we report on the results of experimental and theoretical studies of the Bi-III phase of elemental bismuth. Measurements of the temperature dependence of the magnetic field penetration depth $(\lambda)$ were performed in muon-spin rotation $(\mu \mathrm{SR})$ experiments $(p \simeq$ $2.72 \mathrm{GPa})$. The magnetic field distribution in the sample below $T_{\mathrm{c}}$ reflects the formation of a vortex lattice, thus confirming that Bi-III is a type-II superconductor [17-19]. A zero-temperature value of the magnetic penetration depth $\lambda(0)=301(4) \mathrm{nm}$ was determined. With the coherence length $\xi=10(2) \mathrm{nm}$ taken from the upper critical measurements $[17,18]$, a Ginzburg-Landau parameter $\kappa=30(6)(\kappa=\lambda / \xi)$ was obtained, which turns out to be the highest among known single element superconductors. Density functional theory (DFT) calculations were used to determine the equilibrium lattice structure parameters of Bi-III under pressure. The DFT results of the electronic structure, phonons, and Fermi surfaces are used to analyze different possible scenarios for the onset of superconductivity in Bi-III. Within the framework 

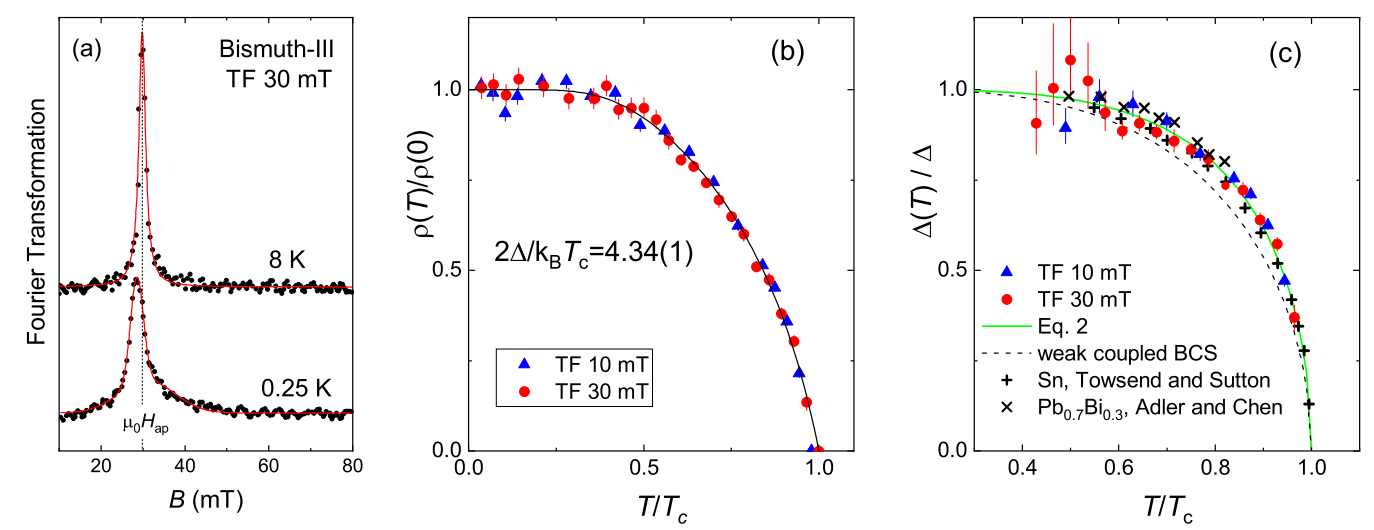

FIG. 1. (a) Fourier transform of TF- $\mu$ SR time spectra $\left(\mu_{0} H_{\text {ap }}=30 \mathrm{mT}\right)$ reflecting the internal field distribution $P(B)$ in the Bi-III sample above $(T=8.0 \mathrm{~K})$ and below $(T=0.25 \mathrm{~K})$ the superconducting transition temperature. The stronger broadening and the asymmetric field distribution in the superconducting state is caused by the formation of a vortex lattice. The solid lines are fits (see Supplemental Material [25]). (b) The temperature dependence of the superfluid density $\rho(T) / \rho(0)=\lambda^{-2}(T) / \lambda^{-2}(0)$ of the Bi-III sample obtained in TF- $\mu$ SR experiments at an applied field $\mu_{0} H_{\text {ap }}=10 \mathrm{mT}$ (blue triangles) and $30 \mathrm{mT}$ (red circles), respectively. The solid line is the fit of Eq. (1) with the gap described by Eq. (2). (c) The temperature dependence of the superconducting gap reconstructed from the superfluid density data. The solid line is the fit of Eq. (2) to the $\Delta(T)$ data. The dashed line is the weakly coupled BCS temperature behavior [43]. The + and $\times$ symbols refer to $\Delta(T) / \Delta$ points for the superconducting $\mathrm{Sn}$ and $\mathrm{Pb}_{0.7} \mathrm{Bi}_{0.3}$ obtained in tunneling experiments (after Townsend and Sutton [44] and Adler and Chen [45]).

of the Eliashberg theory, for a given spectral density of Bi-III, the most effective phonon energy $\omega_{\mathrm{ln}} \simeq 5.5 \mathrm{meV}$ was identified. An alternative pairing mechanism to the electron-phonon coupling which involves the possibility of Cooper pairing induced by Fermi-surface instabilities is also discussed.

The Bi sample was prepared from commercially available Bi granules (Alfa Aesar, 99.997\% purity). The "soft" Bi granules were pressed into a cylinder shape $(\simeq 5.9 \mathrm{~mm}$ in diameter and $\simeq 12 \mathrm{~mm}$ height) and placed inside a double-wall piston-cylinder pressure cell made of MP35/NiCrAl alloy. The construction of the pressure cell is similar to the one described in Refs. [26,46].

The ac susceptibility measurements at $p \simeq 2.72 \mathrm{GPa}$ reveal the presence of a sharp transition with $T_{\mathrm{c}} \simeq 7.05 \mathrm{~K}$ (see Supplemental Material [25]). No indication of a transition at $T \sim 3.9 \mathrm{~K}$ was detected, which would correspond to an admixture of the Bi-II phase as is observed, e.g., in Ref. [17] for pressures up to $2.9 \mathrm{GPa}$.

The transverse-field (TF) $\mu$ SR experiments were carried out at the $\mu \mathrm{E} 1$ beam line by using the dedicated GPD (General Purpose Decay) spectrometer (Paul Scherrer Institute, Switzerland). The details of TF- $\mu$ SR under pressure experiments are provided in the Supplemental Material [25]. Figure 1(a) shows the Fourier transform of TF- $\mu$ SR time spectra (the pressure cell background subtracted, see Supplemental Material [25]) reflecting the internal field distribution $P(B)$ in the Bi-III sample. The asymmetric $P(B)$ distribution at $T \simeq 0.25 \mathrm{~K}$ possesses the basic features expected for an ordered vortex lattice, namely, the cutoff at low fields, the peak shifted below $\mu_{0} H_{\text {ap }}$, and the long tail towards the high-field direction (see, e.g., Refs. [47,48] and references therein). Our experiments confirm, therefore, that the superconductivity of $\mathrm{Bi}-\mathrm{III}$ is of a type-II [17-19].

The temperature dependence of the superfluid density $\left[\rho_{s}(T) / \rho_{s}(T=0)=\lambda^{-2}(T) / \lambda^{-2}(T=0)\right.$, Fig. 1(b) $]$ was further obtained from the second central moment of the magnetic field distribution $\left(\left\langle\Delta B^{2}\right\rangle\right)$. The superconducting part of the second moment, which for extreme type-II superconductors becomes a direct measure of the magnetic penetration depth $\lambda$, was calculated via $\left\langle\Delta B^{2}\right\rangle_{\mathrm{sc}}=\left\langle\Delta B^{2}\right\rangle-\sigma_{\mathrm{nm}}^{2} / \gamma_{\mu}^{2}=$ $0.00371 \Phi_{0}^{2} / \lambda^{4}[49,50]$. Here, $\sigma_{\mathrm{nm}}$ is the contribution of nuclear magnetic moments measured at $T>T_{\mathrm{c}}$ and $\Phi_{0}=$ $2.068 \times 10^{-15} \mathrm{~Wb}$ is the magnetic flux quantum. From the measured $\gamma_{\mu}\left\langle\Delta B^{2}\right\rangle_{\mathrm{sc}}^{1 / 2}(0.25 \mathrm{~K}, 10 \mathrm{mT})=1.22(4) \mu \mathrm{s}^{-1}$ and $\gamma_{\mu}\left\langle\Delta B^{2}\right\rangle_{\mathrm{sc}}^{1 / 2}(0.25 \mathrm{~K}, 30 \mathrm{mT})=1.15(3) \mu \mathrm{s}^{-1}$ the value of the magnetic penetration depth was found to be $\lambda(0.25 \mathrm{~K})=$ 301(4) nm. With the coherence length, $\xi=10(2) \mathrm{nm}$, estimated from the value of the upper critical field $H_{\mathrm{c} 2}(T=0) \simeq$ 2.3-4.6 T [17-19], this translates into a Ginzburg-Landau parameter $\kappa=\lambda / \xi \simeq 30(6)$. To the best of our knowledge this is the highest $\kappa$ value reported to date for known single element superconducting materials. This indicates that Bi-III is a representative of extreme type-II superconductors with $\kappa \gg 1$.

The analysis of the superfluid density data was performed by assuming the superconducting gap of $s$-wave symmetry and using the following functional form [51],

$$
\frac{\rho(T)}{\rho(0)}=1+2 \int_{\Delta(T)}^{\infty}\left(\frac{\partial f}{\partial E}\right) \frac{E d E}{\sqrt{E^{2}-\Delta(T)^{2}}},
$$

where $f=\left[1+\exp \left(E / k_{\mathrm{B}} T\right)\right]^{-1}$ is the Fermi function. The temperature dependence of the superconducting gap $\Delta(T)$ was obtained by solving the nonlinear Eq. (1) for every temperature point. The resulting $\Delta(T) / \Delta$ values for Bi-III are presented in Fig. 1(c). Here, $\Delta$ is the zero-temperature value of the superconducting energy gap. Note that due to saturation of the superfluid density data $[\rho(T) / \rho(0) \simeq 1$ for $T / T_{\mathrm{c}} \lesssim 0.4$, Fig. 1(b)] the low-temperature $\Delta(T)$ values cannot be obtained with reliable accuracy. Figure 1(c) implies that upon decreasing temperature, the gap in Bi-III grows faster than expected for the weakly coupled BCS $\Delta(T)$ [43]. For 
(a)

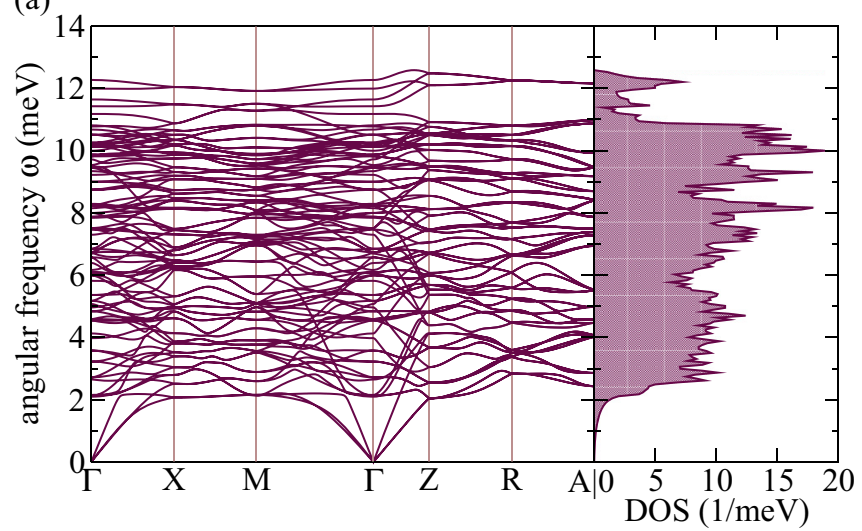

(b)
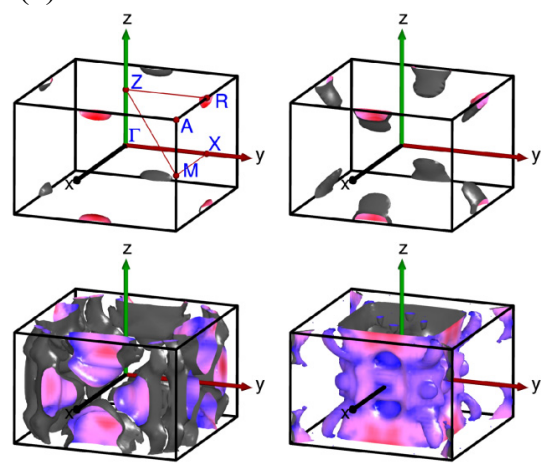
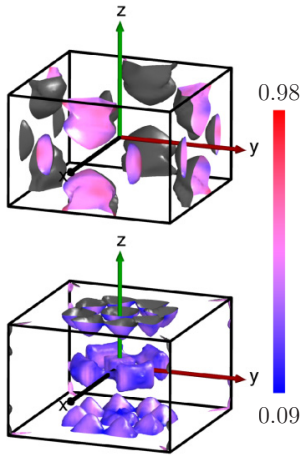

FIG. 2. (a) Phonon dispersion relations and phonon DOS of the Bi-III approximant at $2.7 \mathrm{GPa}$. The logarithmic phonon frequency $\omega_{\mathrm{ln}} \approx$ $6 \mathrm{meV}$ is located in the middle of the phononic band structure. The DOS is normalized to the number of normal modes per unit cell. (b) Nested multisheet Fermi surfaces. The color code indicates the absolute value of the Fermi velocity in units of $10^{6} \mathrm{~m} / \mathrm{s}$. High-symmetry points of the Brillouin zone corresponding to the space group $P 4 / n c c$ in units of $2 \pi\left(\frac{1}{a}, \frac{1}{a}, \frac{1}{c}\right)$. In calculations the approximant of the incommensurate lattice structure of Bi-III by 32 atoms was used.

comparison, the results for the superconducting gap in Sn [44] and $\mathrm{Pb}_{0.7} \mathrm{Bi}_{0.3}$ [45], which were found to be characterized by non-BCS $\Delta(T)$, are also shown in Fig. 1(c). Remarkably, the temperature dependence of the superconducting gap in Sn, $\mathrm{Pb}_{0.7} \mathrm{Bi}_{0.3}$, and $\mathrm{Bi}-\mathrm{III}$ have a very similar functional form.

The temperature dependence of the gap presented in Fig. 1(c) was approximated by the equation [52]

$$
\Delta(T)=\Delta \tanh \left[\frac{\pi k_{\mathrm{B}}}{\Delta} \sqrt{c\left(\frac{T_{\mathrm{c}}}{T}-1\right)}\right],
$$

with $c$ and $\Delta$ as fit parameters. Following Ref. [52] this general equation describes $\Delta(T)$ 's for superconductors with various coupling strengths $\left(2 \Delta / k_{\mathrm{B}} T_{\mathrm{c}}\right)$ and order parameter symmetries. The fit of Eq. (2) to the data with $c=2.31$ (2) and $\Delta=1.32(1) \mathrm{meV}$ is presented in Fig. 1(c) by a solid green line. The solid line in Fig. 1(b) corresponds to the fit of Eq. (1) with such obtained $\Delta(T)$ to the superfluid density data. Using the value of $2 \Delta / k_{\mathrm{B}} T_{\mathrm{c}} \simeq 4.34$ in Carbotte's empirical relation [53],

$$
\frac{2 \Delta}{k_{\mathrm{B}} T_{\mathrm{c}}}=3.53\left[1+12.5\left(\frac{k_{\mathrm{B}} T_{\mathrm{c}}}{\omega_{\ln }}\right)^{2} \ln \frac{\omega_{\ln }}{2 k_{\mathrm{B}} T_{\mathrm{c}}}\right],
$$

the logarithmically averaged phonon frequency $\omega_{\mathrm{ln}} \simeq$ $5.51 \mathrm{meV}$ was calculated. In fact, $\omega_{\mathrm{ln}}$ corresponds to the dynamics of the superconducting state and represents the most effective phonon energy for a given $T_{\mathrm{c}}$. Following the phenomenological arguments of Carbotte [53,54], for Bi-III one expects $\omega_{\text {ln }} \propto 7 k_{\mathrm{B}} T_{\mathrm{c}} \simeq 4.3 \mathrm{meV}$ which is close to $5.51 \mathrm{meV}$ estimated from Eq. (3).

It is worth emphasizing that even though the temperature dependence of the gap in most of the cases was found to follow the weakly coupled BCS prediction, a non-BCS gap behavior is not something that is unexpected. Prominent examples are the metallic $\mathrm{Sn}$ and $\mathrm{Pb}$ [55], in the case of single element superconductors, and $\mathrm{MgB}_{2}$ [56-58] and $\mathrm{PbBi}$ alloys [45] for binary ones. The deviation from weakly coupled BCS $\Delta(T)$ is quite often observed in cuprates and
Fe-based high-temperature superconductors. Theoretically, a non-BCS behavior of $\Delta(T)$ is expected for superconductors with high values of the electron-phonon coupling constant, within the Eliashberg-Nambu formalism [59,60], and is explained by damping of quasiparticle excitations caused by a strong electron-phonon interaction [61-63].

In the following, the results of density functional theory calculations are discussed. The structural analysis and the electronic band structure are presented in the Supplemental Material [25]. The calculations reveal that at ambient pressure, where $\mathrm{Bi}$ adopts a rhombohedral structure (Bi-I phase), its density of states (DOS) shows bands which are clearly separated into bonding/antibonding $s$ and $p$ states. The Fermi level $E_{F}$ is situated in a gap between the bonding and antibonding $p$ states, thus giving rise to the semimetallic behavior of Bi-I [41]. As pressure increases, the $p$ states start to overlap, leading to a metallic DOS at $E_{F}$. From the analysis of electronic bands and DOS (see the Supplemental Material [25]) the host and guest sites in the Bi-III phase were found to have similar magnitudes, in agreement with Ref. [41]. The electronic bands of $p$ character would provide electrons for the pairing.

Figure 2(a) shows the phonon dispersion and the phonon DOS obtained with the approximant of the incommensurate lattice structure of Bi-III by 32 atoms. A different structure of 42 atoms was considered in Ref. [18] and it was found to demonstrate the presence of a low-frequency phason mode. This mode was suggested to be responsible for a very high electron-phonon coupling strength and gave rise to an enhanced transition temperature and a high upper critical field $[18,19]$. Note, however, that in the actual calculations with 32 atoms no phason modes were found to appear in the phonon spectra. One would also mention that in accordance with arguments of the Eliashberg theory, neither the very low nor the very high frequencies are important, while frequencies around the middle of the spectrum, $\approx 6 \mathrm{meV}$ [see Fig. 2(a)], are responsible for the magnitude of $T_{\mathrm{c}}$ [53]. Note that this value stays close to $\omega_{\text {ln }} \simeq 5.51 \mathrm{meV}$ obtained from measured $2 \Delta / k T_{\mathrm{c}} \simeq 4.34$ by means of Eq. (3). The Eliashberg theory provides no arguments on limiting $T_{\mathrm{c}}$ for a given pairing 
mechanism (phonon, phason, or any other boson exchange). Considering the electron-phonon interaction, the limitation on the magnitude of $T_{\mathrm{c}}$ for Bi-III is most probably related to the lattice (in)stability for a given value of the applied pressure.

An alternative superconducting pairing mechanism, based on correlations in the Fermi liquid rather than electron-phonon coupling, was proposed by Kohn and Luttinger [64]. This pairing mechanism is associated with the effective interaction, between quasiparticles occurring as a result of the polarization of the fermionic background, which is proportional to the static susceptibility (Lindhard function). The appearance of a divergence in the static susceptibility $\chi(\mathbf{q})$ is determined by the existence of nesting vectors $\mathbf{q}_{\text {nest }}$ arising if the Fermi surface possesses parallel fragments such that pairs of electronic states can be connected by the same wave vector $\mathbf{q}_{\text {nest }}$. In this case the possibility of Cooper pairing is determined by the characteristics of the energy spectrum (band structure) in the vicinity of the Fermi level and by the effective interaction. In Fig. 2(b) the results of the Fermi-surface (FS) calculations are shown. Based on the FS shape one cannot exclude that such a simple mechanism of Fermi-surface instabilities underlies the pairing formation in $\mathrm{Bi}-\mathrm{III}$, in particular, in conjunction with the Kohn-Luttinger mechanism [64]. Moreover, due to the proximity to a maximum in the DOS, the superconducting transition temperature (predicted within the framework KohnLuttinger mechanics) may increase further as a consequence of a combination with van Hove singularities [65].

To conclude, muon-spin rotation experiments performed under pressure $p \simeq 2.72 \mathrm{GPa}$ indicate that the Bi-III phase of the elemental bismuth is a type-II superconductor characterized by a large Ginzburg-Landau parameter $\kappa=30(6)$. The coupling strength $2 \Delta / k_{\mathrm{B}} T_{\mathrm{c}} \simeq 4.34$ was found, thus implying that $\mathrm{Bi}$-III lies in the strong-coupling regime. The temperature behavior of the superfluid density was found to be consistent with a single gap of $s$-wave symmetry with $\Delta=1.32(1) \mathrm{meV}$. Based on DFT results for the electronic and phononic band structures and Fermi surface, a possible mechanism of superconducting pairing in Bi-III was qualitatively discussed. The DFT results, in combination with the Eliashberg's theory arguments, reveal that phonons with frequency around $5.5 \mathrm{meV}$ are most effective in producing $T_{\mathrm{c}} \approx 7 \mathrm{~K}$. An alternative pairing mechanism to the electron-phonon coupling involves the possibility of Cooper pairing induced by the Fermi-surface instabilities. FS calculations indicate the existence of nesting vectors, so the pairing mechanism of Kohn-Luttinger type may also be considered.

This work was performed at the Swiss Muon Source $(\mathrm{S} \mu \mathrm{S})$, Paul Scherrer Institute (PSI, Switzerland). The work of G.S. is supported by the Swiss National Science Foundation, Grants No. 200021_149486 and No. 200021_175935. L.C. and A.Ö acknowledge DFG for the financial support through TRR80/F6 project. S.S. acknowledges financial support by the Swedish Research Council and computational facilities provided by the Swedish National Infrastructure for Computing at the National Supercomputer Centers in Linköping and Umeå. R.K. is grateful to E. Pomyakushina for providing granular bismuth.
[1] J. Nagamatsu, N. Nakagawa, T. Muranaka, Y. Zenitani, and J. Akimitsu, Nature (London) 410, 63 (2001).

[2] A. P. Drozdov, M. I. Eremets, I. A. Troyan, V. Ksenofontov, and S. I. Shylin, Nature (London) 525, 73 (2015).

[3] V. Struzhkin, M. Eremets, W. Gan, H.-K. Mao, and R. Hemley, Science 298, 1213 (2002).

[4] K. Shimizu, H. Ishikawa, D. Takao, T. Yagi, and K. Amaya, Nature (London) 419, 597 (2002).

[5] S. Deemyad and J. S. Schilling, Phys. Rev. Lett. 91, 167001 (2003).

[6] M. Sakata, Y. Nakamoto, K. Shimizu, T. Matsuoka, and Y. Ohishi, Phys. Rev. B 83, 220512 (2011).

[7] M. Debessai, J. J. Hamlin, and J. S. Schilling, Phys. Rev. B 78, 064519 (2008).

[8] J. Hamlin, V. Tissen, and J. Schilling, Physica C 451, 82 (2007)

[9] M. Ishizuka, M. Iketani, and S. Endo, Phys. Rev. B 61, R3823 (2000).

[10] N. Suzuki and M. Otani, J. Phys.: Condens. Matter 14, 10869 (2002)

[11] V. V. Struzhkin, R. J. Hemley, and H.-K. Mao, Nature (London) 390, 382 (1997).

[12] N. B. Brandt and N. I. Ginzburg, Sov. Phys. JETP 17, 326 (1963)

[13] W. Buckel and J. Wittig, Phys. Lett. 17, 187 (1965).

[14] M. A. Il'ina and E. S. Itskevich, Sov. Phys. JETP Lett. 11, 218 (1970).
[15] N. Lotter and J. Wittig, Europhys. Lett. 6, 659 (1988).

[16] X. Du, S. W. Tsai, D. L. Maslov, and A. F. Hebard, Phys. Rev. Lett. 94, 166601 (2005).

[17] Y. Li, E. Wang, X. Zhu, and H.-H. Wen, Phys. Rev. B 95, 024510 (2017).

[18] P. Brown, K. Semeniuk, D. Wang, B. Monserrat, C. J. Pickard, and F. M. Grosche, Sci. Adv. 4, eaao4793 (2018).

[19] P. Brown, High-pressure states of bismuth, Ph.D. thesis, University of Cambridge, 2017.

[20] M. I. McMahon, O. Degtyareva, and R. J. Nelmes, Phys. Rev. Lett. 85, 4896 (2000).

[21] O. Degtyareva, M. I. MCMahon, and R. J. Nelmes, High Pressure Res. 24, 319 (2004).

[22] J. M. Hastings, J. P. Pouget, G. Shirane, A. J. Heeger, N. D. Miro, and A. G. MacDiarmid, Phys. Rev. Lett. 39, 1484 (1977).

[23] I. U. Heilmann, J. D. Axe, J. M. Hastings, G. Shirane, A. J. Heeger, and A. G. MacDiarmid, Phys. Rev. B 20, 751 (1979).

[24] J. D. Axe and Per Bak, Phys. Rev. B 26, 4963 (1982).

[25] See Supplemental Material at http://link.aps.org/supplemental/ 10.1103/PhysRevB.98.140504 for the details of the experiment and the density functional theory calculations, which includes Refs. [12-19,26-42].

[26] R. Khasanov, Z. Guguchia, A. Maisuradze, D. Andreica, M. Elender, A. Raselli, Z. Shermadini, T. Goko, E. Morenzoni, and A. Amato, High Pressure Res. 36, 140 (2016). 
[27] A. Eiling and J. C. Schilling, J. Phys. F: Met. Phys. 11, 623 (1981).

[28] A. Suter and B. M. Wojek, Phys. Procedia 30, 69 (2012).

[29] R. Khasanov, M. Bendele, A. Amato, K. Conder, H. Keller, H.-H. Klauss, H. Luetkens, and E. Pomjakushina, Phys. Rev. Lett. 104, 087004 (2010).

[30] R. Khasanov, S. Sanna, G. Prando, Z. Shermadini, M. Bendele, A. Amato, P. Carretta, R. De Renzi, J. Karpinski, S. Katrych, H. Luetkens, and N. D. Zhigadlo, Phys. Rev. B 84, 100501(R) (2011).

[31] P. E. Blöchl, Phys. Rev. B 50, 17953 (1994).

[32] G. Kresse and D. Joubert, Phys. Rev. B 59, 1758 (1999).

[33] G. Kresse and J. Furthmüller, Phys. Rev. B 54, 11169 (1996).

[34] J. P. Perdew and Y. Wang, Phys. Rev. B 45, 13244 (1992).

[35] H. J. Monkhorst and J. D. Pack, Phys. Rev. B 13, 5188 (1976).

[36] H. Eschrig, M. Richter, and I. Opahle, in Relativistic Electronic Structure Theory, Part 2: Applications, edited by P. Schwerdtfeger (Elsevier, Amsterdam, 2004), Chap. 12, pp. 723-776.

[37] K. Koepernik and H. Eschrig, Phys. Rev. B 59, 1743 (1999).

[38] G. Lehmann and M. Taut, Phys. Status Solidi B 54, 469 (1972).

[39] P. E. Blöchl, O. Jepsen, and O. K. Andersen, Phys. Rev. B 49, 16223 (1994).

[40] A. Togo and I. Tanaka, Scr. Mater. 108, 1 (2015).

[41] U. Häussermann, K. Söderberg, and R. Norrestam, J. Am. Chem. Soc. 124, 15359 (2002).

[42] H. T. Stokes and D. M. Hatch, FINDSYM (2004), http://stokes.byu.edu/isotropy.html [J. Appl. Crystallogr. 38, 237 (2005)].

[43] B. Mühlschlegel, Z. Phys. 155, 313 (1959).

[44] P. Townsend and J. Sutton, Phys. Rev. 128, 591 (1962).

[45] J. G. Adler and T. T. Chen, Solid. State. Commun. 9, 1961 (1971).

[46] Z. Shermadini, R. Khasanov, M. Elender, G. Simutis, Z. Guguchia, K. V. Kamenev, and A. Amato, High Pressure Res. 37, 449 (2017).
[47] A. Maisuradze, R. Khasanov, A. Shengelaya, and H. Keller, J. Phys.: Condens. Matter 21, 075701 (2009).

[48] R. Khasanov, H. Zhou, A. Amato, Z. Guguchia, E. Morenzoni, X. Dong, G. Zhang, and Z.-X. Zhao, Phys. Rev. B 93, 224512 (2016).

[49] E. H. Brandt, Phys. Rev. B 37, 2349(R) (1988).

[50] E. H. Brandt, Phys. Rev. B 68, 054506 (2003).

[51] M. Tinkham, Introduction to Superconductivity (Krieger, Malabar, FL, 1975).

[52] R. Prozorov, Supercond. Sci. Technol. 21, 082003 (2008).

[53] J. P. Carbotte, Rev. Mod. Phys. 62, 1027 (1990).

[54] J. M. Coombes and J. P. Carbotte, Phys. Rev. B 38, 8697 (1988).

[55] R. F. Gasparovic, B. N. Taylor, and R. E. Eck, Solid State Commun. 4, 59 (1966).

[56] S. Lee, Z. G. Khim, Y. Chong, S. H. Moon, H. N. Lee, H. G. Kim, B. Oh, and E. J. Choi, Physica C 377, 202 (2002).

[57] Y. G. Ponomarev, S. A. Kuzmichev, M. G. Mikheev, M. V. Sudakova, S. N. Tchesnokov, N. Z. Timergaleev, A. V. Yarigin, E. G. Maksimov, S. I. Krasnosvobodtsev, A. V. Varlashkin, M. A. Hein, G. Müller, H. Piel, L. G. Sevastyanova, O. V. Kravchenko, K. P. Burdina, and B. M. Bulychev, Solid State Commun. 129, 85 (2004).

[58] A. Brinkman, A. A. Golubov, H. Rogalla, O. V. Dolgov, J. Kortus, Y. Kong, O. Jepsen, and O. K. Andersen, Phys. Rev. B 65, 180517(R) (2002).

[59] C. R. Leavens, Phys. Rev. B 29, 5178 (1984).

[60] X. H. Zheng and D. G. Walmsley, Phys. Rev. B 77, 104510 (2008).

[61] T. R. Schrieffer and Y. Wada, Bull. Am. Phys. Soc. 8, 307 (1963).

[62] Y. Wada, Rev. Mod. Phys. 36, 253 (1964).

[63] Y. Wada, Phys. Rev. 135, A1481 (1964).

[64] W. Kohn and J. M. Luttinger, Phys. Rev. Lett. 15, 524 (1965).

[65] R. Hlubina, Phys. Rev. B 59, 9600 (1999). 